\title{
Immunology Taught by Bacteria
}

\author{
Russell E. Vance
}

Received: 11 March 2010 /Accepted: 12 March 2010 / Published online: 6 April 2010

(C) The Author(s) 2010. This article is published with open access at Springerlink.com

\begin{abstract}
Introduction It has been proposed that the innate immune system might discriminate living and virulent pathogens from dead or harmless microbes, but the molecular mechanisms by which this discrimination could occur remain unclear. Although studies of model antigens and adjuvants have illuminated important principles underlying immune responses, the specific immune responses made to living, virulent pathogens can only be discovered by studies of the living, virulent pathogens themselves.

Methods and Findings Here, I review what one particular bacterium, Legionella pneumophila, has taught us about the innate immune response. Pathogens differ greatly in the mechanisms they use to invade, replicate within, and transmit among their hosts. However, a theme that emerges is that the pathogenic activities sensed by host cells are conserved among multiple pathogenic bacteria.

Conclusion Thus, immunology taught by L. pneumophila may lead to a more general understanding of the host response to infection.
\end{abstract}

Keywords Legionella pneumophila $\cdot$ inflammasome · innate immunity · flagellin · interferon

In 1996, Rolf Zinkernagel published a review in Science entitled "Immunology Taught by Viruses" [1]. Zinkernagel's message was targeted to a generation of immunologists that had, since Landsteiner [2], focused on dissecting

R. E. Vance $(\bowtie)$

Division of Immunology \& Pathogenesis, Department of Molecular \& Cell Biology,

University of California, Berkeley,

415 Life Science Addition,

Berkeley, CA 94720, USA

e-mail: rvance@berkeley.edu immune responses to model antigens such as haptens or ovalbumin. While the use of model antigens has been essential for deciphering the basic mechanisms of adaptive immunity, Zinkernagel rightly argued that a deeper appreciation for viruses could provide new insights. Since the continued existence of viruses depends on their ability to evade the immune systems of their hosts, there may be no stronger evidence for the importance of a given immune function than a demonstration that a virus has evolved a mechanism to evade it. In urging immunologists to study viruses, Zinkernagel echoed Janeway's prior assertion that progress in immunology "will require a rediscovery of microbiology by immunologists" [3]. Certainly, the ensuing two decades of work in innate immunity, including the discovery of Toll-like and other innate receptors, has demonstrated how immunology can learn from microbiology.

Although viruses may be great teachers, they are also pedagogically problematic in many ways. Since viruses are highly co-evolved with their hosts, it is sometimes difficult to be certain whether an observed immune response to a virus is beneficial to the host or the virus. The evasion of many immune responses by viruses means that important immune responses are not always readily apparent or easy to study. And, although viral evasion of an immune response is strong evidence for the importance of that immune response, there is always the nagging worry that any immune response that can be easily evaded might not really be that important after all. Lastly, viruses are just one class of microbe that can cause disease, and it is unlikely that all the principles of immunity gleaned from viral infections will apply to other types of infection. Indeed, there is still much to learn about the fundamental biology of the immune response, and different infectious microbes can teach us different things. In this review, I illustrate how 
bacteria can teach us immunology by focusing on our recent progress in understanding the innate immune pathways that recognize Legionella pneumophila.

\section{The Teacher: L. pneumophila}

L. pneumophila is a gram-negative motile bacterium that causes a severe pneumonia called Legionnaires' disease. Legionella infection is typically acquired by humans through inhalation of a large dose of aerosolized bacteria, frequently arising from bacterial contamination of a cooling tower associated with a building's air-conditioning system. This mode of infection appears to be the explanation for the notorious outbreak of Legionella infections at the 1976 Legionnaires' Convention in Philadelphia, as well as several subsequent outbreaks. Once inhaled, Legionella is believed to replicate primarily in alveolar macrophages in the lung, though other cell types may also be targeted. Although Legionella infection can result in significant human morbidity and mortality, Legionella's real "purpose" in life has little to do with us. Through most of its evolution, Legionella's natural history appears to have occurred primarily in various species of freshwater amoebae. Humans are believed to be "accidental" and dead-end hosts, mere victims of "progress" that has come in the form of the recent invention of large-scale air-conditioning systems. Legionella does not appear to be transmitted from human to human [4], or even animal to animal [5], leading to the view that Legionella does not evolve in mammals and has thus probably not evolved mechanisms to evade mammalian immune responses.

The ability of $L$. pneumophila to infect macrophages appears to derive from the fundamentally conserved cell biology of amoebae and macrophages [6]. Replication of L. pneumophila in amoebae and in macrophages occurs within a membrane-bound compartment called the Legionella-containing vacuole (LCV). The creation of the LCV by L. pneumophila requires a bacterial type IV secretion system called the Icm/Dot system, a macromolecular channel that inserts in the phagosomal membrane and delivers bacterial proteins, termed effectors, into the host cell cytosol. Well over $150 \mathrm{~L}$. pneumophila effectors are believed to be translocated by the Icm/Dot system into the host cell cytosol, where they carry out diverse functions. There is evidence that the Icm/Dot system can also translocate nucleic acids [7] and probably fragments of peptidoglycan as well [8]. The primary function of the Icm/Dot-translocated protein effectors appears to be creation of the LCV. I $\mathrm{cm} /$ Dot-deficient mutants are unable to replicate in host cells and are instead rapidly targeted to a compartment harboring late-endosomal markers such as LAMP1. Consistent with these observations, several translocated effectors have been shown to regulate vesicular traffic in infected cells and recruit membrane to the L. pneumophila-containing phagosome [6]. However, other translocated effectors appear to have functions unrelated to vesicular traffic. For example, a small family of translocated effectors functions to inhibit host protein synthesis, whereas other translocated effectors appear to function to inhibit host cell death.

Amoebae and vertebrates are separated by at least a billion years of evolution. Given that many intracellular pathogens exhibit relatively restricted host ranges, it is surprising that there is sufficient conservation of biology to allow such a dramatic jump of host species. For this reason, it is possible that the adaptability of L. pneumophila is explained by infection of cryptic vertebrate hosts in the environment. Vertebrate hosts that can sustain transmission of L. pneumophila have not yet been found, however, and there is no strong evidence that $L$. pneumophila is at all immune evasive. Although amoebae certainly have some primitive immune defense mechanisms, immunity in amoebae obviously does not resemble the comparatively sophisticated multicellular innate and adaptive immune systems present in vertebrates. Thus, L. pneumophila can be thought of as a highly educated cell biologist, but also as an ignorant immunologist.

Why would an immunologist want to study such an immunologically unsophisticated pathogen? As is borne out by a few examples discussed briefly below, it appears that one of the advantages of studying L. pneumophila is that it provokes robust host responses-responses that would likely have to be suppressed by better-adapted pathogens. Moreover, since L. pneumophila is not closely related to any other bacterial species that have been the subject of extensive immunological analysis, we have reason to believe that $L$. pneumophila may be able to teach us new things. A third reason for learning from L. pneumophila is that just as L. pneumophila is not adapted to humans, nor is it likely that the human immune system has evolved specifically to recognize $L$. pneumophila. The selective pressure that has driven mammals to respond vigorously to L. pneumophila has likely come not from L. pneumophila itself, but from other pathogens that have made a more significant imprint on our evolutionary history. Thus, whatever we learn from L. pneumophila is not likely to be relevant only to one bacterial species, but should allow us to discern broader principles of immune recognition.

\section{Patterns of Pathogenesis: Immunology Taught by Living Pathogens}

Since Janeway's exhortation to rediscover microbiology [3], immunologists have done just that, dissecting innate responses to microbial ligands such as lipopolysaccharide 
or flagellin. Janeway referred to these stimulatory molecules as pathogen-associated microbial patterns (PAMPs), and the discovery of Toll-like receptors (TLRs) and other innate receptors has resoundingly vindicated Janeway's prediction that the host would express germline-encoded receptors that recognize conserved microbial molecules. TLRs were discovered primarily by the use of purified PAMPs or killed microbes, but of course, pathogens are more than just a "bag of PAMPs", and so a recent goal has been to extend the vision of Janeway to the study of innate responses to living, virulent microbes. Our hypothesis is that, in addition to sensing of pathogen-encoded molecules (PAMPs), hosts might also sense particular pathogenencoded activities that we have termed "patterns of pathogenesis" [9]. Despite the diversity of pathogens, we posit that there may be a few common virulence-related activities - for example, cytosolic invasion or disruption of actin - that the immune system could detect in order to trigger specific host responses. Our hypothesis resembles a longstanding hypothesis from the plant immunity literature called the Guard Hypothesis [10], but few examples of guard-type mechanisms have been shown to occur in mammals.

Wild-type and isogenic Icm/Dot-deficient L. pneumophila mutants provide a simple genetic model for comparing infections with virulent and avirulent microbes. A theme that has emerged from this model is that, although the Icm/Dot system is essential for L. pneumophila replication, Icm/Dot expression is also associated with induction of several robust innate immune responses, some of which act to restrict bacterial replication. Below, I briefly discuss several independent immune surveillance pathways stimulated specifically by virulent $\mathrm{Icm} / \mathrm{Dot}^{+}$ bacteria.

\section{Cytosolic Sensing of Flagellin by the Naip5/Ipaf Inflammasome}

More than 20 years ago, Friedman and colleagues discovered that macrophages from the $\mathrm{A} / \mathrm{J}$ strain of mouse were several logs more permissive to $L$. pneumophila replication than were macrophages from other strains, including C57BL/6 [11]. Years of genetic analysis traced the resistance of C57BL/6 to the function of a single genetic locus, $\operatorname{Lgn} 1$, and ultimately to a single gene, Naip 5 , within this locus $[12,13]$. Naip 5 bears homology to other genes such as Nodl and Nod2 that had previously been shown to function as cytosolic sensors of different fragments of peptidoglycan. Thus, the molecular cloning of Naip5 led to the immediate hypothesis that Naip5 detects a $L$. pneumophila-derived ligand, leading to induction of an anti-bacterial immune response. However, the nature of the ligand and the ensuing response were far from clear.
Nod1 and Nod2 both contain caspase activation and recruitment domains (CARDs) that recruit the same downstream kinase, Rip2, leading to $N F-\kappa B$ activation and transcriptional responses. By contrast, Naip5 lacks CARDs; moreover, Naip5 does not seem to activate $\mathrm{NF}-\mathrm{kB}$, and, in our hands, at least, does not appear to regulate significant transcriptional responses (though others report modest transcriptional effects [14]). Several studies have demonstrated that a functional Naip 5 allele correlates with altered maturation of $L$. pneumophila-containing phagosome [15-17], but there is no direct evidence these observed maturation differences are causally responsible for the observed restriction of bacterial replication. Nor is there a clear molecular understanding of how Naip 5 regulates the phagosomal maturation process. We tend to favor the idea that host cell suicide, rather than altered phagosome trafficking, is primarily responsible for restriction of bacterial replication. Since L. pneumophila requires a host cell niche in which to replicate, rapid host cell death would be expected to curtail bacterial replication. This model originated from studies showing that a functional Naip5 allele (from the C57BL/6 strain) correlated with increased cell death [18] and caspase-1 activation [19] in response to L. pneumophila infection. Evidence against the host cell death model has come from studies showing that permissive macrophages from the $\mathrm{A} / \mathrm{J}$ strain of macrophage appear to activate caspase- 1 and die as efficiently as restrictive C57BL/6 macrophages [20]. However, other reports using quantitative single cell assays [21, 22] have observed differences in host cell death between $\mathrm{A} / \mathrm{J}$ and $\mathrm{C} 57 \mathrm{BL} / 6$ macrophages. Moreover, consistent with the cell death model, macrophages from Naip5-deficient mice are permissive to L. pneumophila and fail to activate caspase-1 or die in response to L. pneumophila infection [23]. Nevertheless, future studies are necessary to resolve the signaling events that occur downstream of Naip5.

By contrast, the signaling events upstream of Naip5 are better understood. A breakthrough was the finding that flagellin-deficient mutants of $L$. pneumophila evade Naip5-mediated restriction and fail to activate caspase-1dependent cell death, leading to the hypothesis that the cytosolic presence of flagellin activates Naip5 [22, 24]. These studies were the first to suggest that host cells survey the cytosol for the presence of flagellin. It was also shown that a cytosolic surveillance pathway detected cytosolic flagellin delivered by Salmonella $[25,26]$. In the case of Salmonella, a type III secretion system delivers flagellin to the cytosol, whereas for L. pneumophila, the Icm/Dot type IV secretion system is the relevant translocon. Subsequent studies demonstrated conclusively that the $\mathrm{C}$-terminus of flagellin is a sufficient signal to activate Naip5 and caspase-1 [23]. Naip5 appears to function with its paralog, Ipaf, which is also essential for detection of 
flagellin and restriction of $L$. pneumophila replication $[19,25,26]$.

Toll-like receptor 5 (TLR5) is a cell surface receptor that also recognizes flagellin. Notably, Naip5/Ipaf-dependent recognition of flagellin does not involve TLR5 [22, 24-26], and in fact, the two sensor systems respond to distinct parts of the flagellin molecule [23]. Why would host cells have evolved separate cytosolic and cell-surface detection systems for flagellin? We suspect that each flagellin-sensing system has its purpose and that the presence of flagellin in the cytosol is interpreted by the host as signifying a different and more serious threat than the presence of extracellular flagellin, which could result from transient bacteremia with harmless commensal bacteria. Flagellin can only reach the cytosol if delivered there by a microbe with a secretion system, and such microbes are almost certain to be pathogens. In fact, although it is a PAMP (flagellin) that is sensed by Naip5/Ipaf, it seems likely that flagellin serves only as a proxy - its detection is simply a way to detect a pathogen-associated activity, namely secretion of bacterial molecules into the cytosol.

\section{Transcriptional Responses to Virulent $L$. pneumophila}

Genetic elimination of the flagellin-sensing pathway by use of flagellin-deficient $L$. pneumophila mutants, or Naip5/Ipaf knockout mice, has little effect on the transcriptional responses made specifically to virulent $L$. pneumophila. For example, one prominent transcriptional response to $L$. pneumophila involves the induction of the cytokine interferon- $\beta$. This response requires bacterial expression of the Icm/Dot system, but is independent of Toll-like receptors, flagellin, Naip5, or Ipaf [27]. Instead, induction of type I IFN appears to involve, at least in part, the cytosolic RNA sensors RIG-I and/or MDA5 [28-30]. A role for RIG-I/MDA5 was unexpected, since cytosolic RNA sensors were previously thought only to detect viruses. The mechanism by which RIG-I and/or MDA5 are activated by L. pneumophila remains uncertain [29]. Regardless, the observed RIG-I-dependent induction of type I IFNs only occurs upon infection with live, virulent $L$. pneumophila and would not have been discovered using purified PAMPs or killed bacteria.

Cytosolic RNA sensors do not appear to be the only transcriptional pathways induced by virulent $L$. pneumophila. In addition, Icm/Dot-dependent activation of NF- $\mathrm{KB}$ downstream of Nod1 and Nod2 has been observed [8], presumably in response to translocated fragments of peptidoglycan. There also appears to be an Icm/Dotdependent TLR/Nod1/Nod2/flagellin-independent pathway leading to induction of MAP kinases and several important inflammatory cytokines [8]. The molecular basis for this novel pathway is still uncertain, but it seems to be a specific host response to virulent $L$. pneumophila.

\section{Conclusion}

Studies with L. pneumophila illustrate the important point that pathogenic and non-pathogenic microbes can be discriminated by the innate immune system. In the cases discussed above, the sensors involved in pathogen-specific responses are not TLRs, but are instead a variety of cytosolic sensors that are selectively activated in response to virulent $\mathrm{Icm} / \mathrm{Dot}^{+} L$. pneumophila that accesses the cytosol as part of its virulence strategy. In other infection scenarios, TLRs may also be activated specifically in response to pathogenic microbes. For example, selective basolateral expression of TLRs might permit responses to occur only upon infection with microbes capable of disrupting epithelial barriers. It is clear that pathogens employ diverse mechanisms of pathogenesis and each pathogen is likely to provoke a distinct set of host responses. Regardless of what might also be learned from studies of other living virulent pathogens, L. pneumophila has proven to be a powerful tool for discovery of several cytosolic immunosurveillance pathways and serves as an example of how immunology can be taught by bacteria.

Acknowledgements I acknowledge collaborators past and present, especially those that have contributed directly to the work I have discussed, including S. Banga, S. Brubaker, J. Coers, W. Dietrich, E. Dunipace, M. Fontana, K. Lightfield, Z.-Q. Luo, S. McWhirter, K. Monroe, J. Persson, T. Ren, C. Roy, J. von Moltke, and D. Zamboni. I am also grateful for stimulating discussions with G. Barton, R. Isberg, and D. Portnoy. I gratefully acknowledge the financial support of the NIH (AI075039, AI082357, and AI082357), the Cancer Research Institute, and the Burroughs Wellcome Fund.

Open Access This article is distributed under the terms of the Creative Commons Attribution Noncommercial License which permits any noncommercial use, distribution, and reproduction in any medium, provided the original author(s) and source are credited.

\section{References}

1. Zinkernagel RM. Immunology taught by viruses. Science. 1996;271:173-8.

2. Landsteiner K. The specificity of serological reactions. Cambridge, MA: Harvard University Press; 1945.

3. Janeway Jr CA. Approaching the asymptote? Evolution and revolution in immunology. Cold Spring Harbor Symp Quant Biol. 1989;54(Pt 1):1-13.

4. Fraser DW, Tsai TR, Orenstein W, et al. Legionnaires' disease: description of an epidemic of pneumonia. N Engl J Med. 1977;297:1189-97. 
5. Katz SM, Habib WA, Hammel JM, et al. Lack of airborne spread of infection by Legionella pneumophila among guinea pigs. Infect Immun. 1982;38:620-2.

6. Isberg RR, O'Connor TJ, Heidtman M. The Legionella pneumophila replication vacuole: making a cosy niche inside host cells. Nat Rev Microbiol. 2009;7:13-24.

7. Vogel JP, Andrews HL, Wong SK, et al. Conjugative transfer by the virulence system of Legionella pneumophila. Science. 1998;279:873-6.

8. Shin S, Case CL, Archer KA, et al. Type IV secretion-dependent activation of host MAP kinases induces an increased proinflammatory cytokine response to Legionella pneumophila. PLoS Pathog. 2008;4:e1000220.

9. Vance RE, Isberg RR, Portnoy DA. Patterns of pathogenesis: discrimination of pathogenic and nonpathogenic microbes by the innate immune system. Cell Host Microbe. 2009;6:10-21.

10. Dangl JL, Jones JD. Plant pathogens and integrated defence responses to infection. Nature. 2001;411:826-33.

11. Yamamoto Y, Klein TW, Newton CA, et al. Growth of Legionella pneumophila in thioglycolate-elicited peritoneal macrophages from A/J mice. Infect Immun. 1988;56:370-5.

12. Diez E, Lee SH, Gauthier S, et al. Bircle is the gene within the Lgn1 locus associated with resistance to Legionella pneumophila. Nat Genet. 2003;33:55-60.

13. Wright EK, Goodart SA, Growney JD, et al. Naip5 affects host susceptibility to the intracellular pathogen Legionella pneumophila. Curr Biol. 2003;13:27-36.

14. Fortier A, Doiron K, Saleh M, et al. Restriction of Legionella pneumophila replication in macrophages requires concerted action of the transcriptional regulators Irf1 and Irf8 and nod-like receptors Naip5 and Nlrc4. Infect Immun. 2009;77:4794-805.

15. Amer A, Franchi L, Kanneganti TD, et al. Regulation of Legionella phagosome maturation and infection through flagellin and host IPAF. J Biol Chem. 2006;281:35217-23.

16. Fortier A, de Chastellier C, Balor S, et al. Birc1e/Naip5 rapidly antagonizes modulation of phagosome maturation by Legionella pneumophila. Cell Microbiol. 2006;9:910-23.

17. Watarai M, Derre I, Kirby J, et al. Legionella pneumophila is internalized by a macropinocytotic uptake pathway controlled by the Dot/Icm system and the mouse Lgn1 locus. J Exp Med. 2001;194:1081-96.
18. Derre I, Isberg RR. Macrophages from mice with the restrictive Lgn1 allele exhibit multifactorial resistance to Legionella pneumophila. Infect Immun. 2004;72:6221-9.

19. Zamboni DS, Kobayashi KS, Kohlsdorf T, et al. The Bircle cytosolic pattern-recognition receptor contributes to the detection and control of Legionella pneumophila infection. Nat Immunol. 2006;7:318-25.

20. Lamkanfi M, Amer A, Kanneganti TD, et al. The nod-like receptor family member Naip5/Bircle restricts Legionella pneumophila growth independently of caspase-1 activation. J Immunol. 2007;178:8022-7.

21. Derre I, Isberg RR. Legionella pneumophila replication vacuole formation involves rapid recruitment of proteins of the early secretory system. Infect Immun. 2004;72:3048-53.

22. Ren T, Zamboni DS, Roy CR, et al. Flagellin-deficient Legionella mutants evade caspase-1- and Naip5-mediated macrophage immunity. PLoS Pathog. 2006;2:e18.

23. Lightfield KL, Persson J, Brubaker SW, et al. Critical function for Naip5 in inflammasome activation by a conserved carboxyterminal domain of flagellin. Nat Immunol. 2008;9:1171-8.

24. Molofsky AB, Byrne BG, Whitfield NN, et al. Cytosolic recognition of flagellin by mouse macrophages restricts Legionella pneumophila infection. J Exp Med. 2006;203:1093-104.

25. Franchi L, Amer A, Body-Malapel M, et al. Cytosolic flagellin requires Ipaf for activation of caspase-1 and interleukin 1beta in salmonella-infected macrophages. Nat Immunol. 2006;7:576-82.

26. Miao EA, Alpuche-Aranda CM, Dors M, et al. Cytoplasmic flagellin activates caspase-1 and secretion of interleukin 1 beta via Ipaf. Nat Immunol. 2006;7:569-75.

27. Stetson DB, Medzhitov R. Recognition of cytosolic DNA activates an IRF3-dependent innate immune response. Immunity. 2006;24:93-103.

28. Chiu YH, Macmillan JB, Chen ZJ. RNA polymerase III detects cytosolic DNA and induces type I interferons through the RIG-I pathway. Cell. 2009;138:576-91.

29. Monroe KM, McWhirter SM, Vance RE. Identification of host cytosolic sensors and bacterial factors regulating the type I interferon response to Legionella pneumophila. PLoS Pathog. 2009;5: e1000665.

30. Opitz B, Vinzing M, van Laak V, et al. Legionella pneumophila induced IFNbeta in lung epithelial cells via IPS-1 and IRF3 which also control bacterial replication. J Biol Chem. 2006;281:36173-9. 